Port Acadie

Revue interdisciplinaire en études acadiennes

An Interdisciplinary Review in Acadian Studies

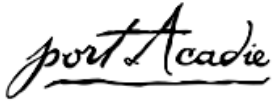

\title{
Actualité artistique et réception immédiate : les enjeux de la critique en Acadie
}

\section{Ariane Brun del Re et Pénélope Cormier}

Numéro 27, printemps 2015

URI : https://id.erudit.org/iderudit/1038346ar

DOI : https://doi.org/10.7202/1038346ar

Aller au sommaire du numéro

\section{Éditeur(s)}

Université Sainte-Anne

ISSN

1498-7651 (imprimé)

1916-7334 (numérique)

Découvrir la revue

Citer cet article

Brun del Re, A. \& Cormier, P. (2015). Actualité artistique et réception immédiate : les enjeux de la critique en Acadie. Port Acadie, (27), 27-46. https://doi.org/10.7202/1038346ar
Résumé de l'article

Prenant comme point de départ la fermeture de l'espace dédié à la critique des arts acadiens « Tintamarre » du journal L'Acadie Nouvelle en 2013, cet article présente les enjeux épistémologiques de la réception immédiate comme discours sur les arts. À partir d'un historique et d'un état des lieux de la critique artistique en Acadie, il débusque certaines idées préconçues sur les défis et les obstacles de la critique artistique en milieu minoritaire

- concernant notamment l'absence de volontaires, leur formation et leurs conditions de travail, mais aussi leur rôle et les réactions des lecteurs - en situant le discours critique acadien comme le maillon faible de la chaîne de production culturelle. 


\title{
Actualité artistique et réception immédiate : les enjeux de la critique en Acadie
}

\author{
Ariane Brun del Re, Université \\ d'Ottawa \\ Pénélope Cormier, Université de \\ Moncton, campus d'Edmundston
}

\begin{abstract}
Résumé
Prenant comme point de départ la fermeture de l'espace dédié à la critique des arts acadiens «Tintamarre » du journal L'Acadie Nouvelle en 2013, cet article présente les enjeux épistémologiques de la réception immédiate comme discours sur les arts. À partir d'un historique et d'un état des lieux de la critique artistique en Acadie, il débusque certaines idées préconçues sur les défis et les obstacles de la critique artistique en milieu minoritaire - concernant notamment l'absence de volontaires, leur formation et leurs conditions de travail, mais aussi leur rôle et les réactions des lecteurs - en situant le discours critique acadien comme le maillon faible de la chaîne de production culturelle.
\end{abstract}

\section{Introduction}

À l'automne 2013, le journal L'Acadie Nouvelle cessait la publication de "Tintamarre ", son espace hebdomadaire de critique des arts acadiens, tenu par David Lonergan depuis 1994. Bien que cette décision n'ait pas sonné le glas de la critique artistique sur les arts acadiens, qui se poursuit ailleurs et par diverses plumes ${ }^{1}$, il n'empêche que la perte de la plus en vue des tribunes de la critique artistique - publiée en Acadie, avec régularité et continuité, sur support papier et par l'un des principaux médias acadiens - a été un choc pour la communauté artistique. S'ajoutant à la fermeture des Éditions d'Acadie en 2000 et de la revue de création éloizes ${ }^{2}$ deux ans plus tard, cette disparition rappelle à tous la fragilité des institutions artistiques acadiennes. Par ailleurs, lors d'une conférence de 2011, soit avant même l'abolition de "Tintamarre ", Raoul Boudreau établissait un bilan alarmiste de la critique littéraire en Acadie, estimant qu'avec l'édition, l'« autre domaine dans lequel l'institution

1. En 2005 , le périodique trimestriel Liaison a élargi son mandat franco-ontarien à la couverture de l'actualité artistique franco-canadienne; à ce jour, cette revue demeure la principale source de critiques des arts de l'Acadie, de l'Ontario français et de l'ouest francophone. Les revues québécoises Nuit blanche et Lettres québécoises publient assez fréquemment des critiques d'œuvres littéraires acadiennes. D'autres médias acadiens et québécois font une place à la critique des arts acadiens, quoique pas de façon régulière ni continue. Depuis l'automne 2014, le webzine acadien Astheure dispose d'une section de critique artistique, coéditée par Ariane Brun del Re et Pénélope Cormier.

2. L'arrêt d'éloizes était déjà une perte pour la critique artistique en Acadie, puisque la revue comprenait une section de réception des œuvres littéraires récentes. 
littéraire acadienne peine à établir des structures solides est celui de la réception immédiate des œuvres, c'est-à-dire les recensions et comptes rendus dans les journaux et les revues, à la radio ou à la télévision »3.

La critique artistique serait-elle donc le maillon le plus faible de la chaîne de production culturelle en Acadie 4 ? Il est vrai que l'investissement dans la critique et dans le processus d'institutionnalisation de l'art en Acadie n'a peut-être pas été suffisant; comme le note encore Boudreau, l'Acadie ne se serait pas donné les moyens de ses ambitions :

Comme pour les besoins d'édition qu'elle n'a pu combler elle-même, l'Acadie s'est tournée vers l'Ontario et le Québec pour fournir une réception immédiate de ses œuvres littéraires.

[...] Comment concilier ce recours aux services des voisins avec l'aspiration à une plus grande autonomie de la littérature acadienne? ${ }^{5}$

Se remémorant le contexte entourant la mise sur pied des premières institutions culturelles en Acadie, dans les années 1970, l'artiste multidisciplinaire Herménégilde Chiasson explique que les créateurs acadiens, à ce moment de l'institutionnalisation des arts en Acadie, sont "des hommes- et des femmes-orchestres », qui doivent
à la fois créer les infrastructures (galeries d'art, maisons d'édition, compagnie de théâtre) [et] les alimenter en produisant les œuvres, se faire promoteurs, graphistes, critiques, collectionneurs, spectateurs et pouvoir se justifier auprès des instances gouvernementales et des bailleurs de fonds. ${ }^{6}$

En Acadie, quarante ans plus tard, force est de constater que la réception immédiate ne s'est pas institutionnalisée au même rythme ni avec la même durabilité que le reste de la chaîne de production culturelle que

3. Raoul Boudreau, "L'institution littéraire acadienne : une étoile qui s'étiole? », dans Benoit Doyon-Gosselin, David Bélanger et Cassie Bérard (dir.), Les institutions littéraires en question dans la Franco-Amérique, Québec, Presses de l'Université Laval, coll. «Culture française d’Amérique », 2014, p. 18.

4. Les commentateurs s'entendent en général pour dire que l'exiguïté du public est le défi le plus important des arts acadiens. Certes, le nombre absolu de consommateurs potentiels d'art est un facteur objectif, limité par la démographie. Cependant, on peut postuler que le développement de la critique artistique et le développement d'un public averti sont liés.

5. Raoul Boudreau, op. cit., p. 18.

6. Herménégilde Chiasson, "Trajectoire et nostalgie », dans Neue Romania, $\mathrm{n}^{\circ}$ 29, 2004 , p. 41. 
trace ici Chiasson. Or, selon Jean-Marie Klinkenberg, le développement d'outils de réception parachève le processus de formation d'un espace artistique. En effet, à la suite des critères d'autonomisation d'un espace littéraire - lectorat suffisant et homogène, nombre critique d'œuvres se réclamant également d'une certaine homogénéité, œuvres reconnues comme fondatrices et reconnaissance de la légitimité du corpus ${ }^{7}-$, il indique que "[l]e couronnement du processus est l'institutionnalisation proprement dite : appareils de légitimation et de consécration ${ }^{8}$, dont font partie, entre autres, les prix littéraires, la critique et l'enseignement.

Les analyses institutionnelles de l'art en Acadie ont peu examiné la critique artistique. Parmi les études qui s’intéressent aux enjeux institutionnels de la réception, des articles abordent l'enseignement ${ }^{9}$, se penchent sur les querelles littéraires ${ }^{10}$ ou encore sur les prix littéraires ${ }^{11}$,

7. Voir Jean-Marie Klinkenberg, «Introduction - L'analyse institutionnelle de la littérature en Belgique francophone : où en est-on? ", Textyles, $n^{\circ} 15,1999$, § 7; sur Internet : http://textyles.revues.org/1021 (page consultée le 28 décembre 2015).

8. Ibid.

9. Voir Raoul Boudreau, « L'institutionnalisation inachevée de la littérature acadienne ", dans Janine Gallant, Hélène Destrempes et Jean Morency (dir.), L'œuvre littéraire et ses inachèvements, Longueuil (Québec), Groupéditions, 2007, p. 153-167; James De Finney, Carole Boucher et David Lonergan, « L'institution littéraire acadienne : ouverture et pluralisation », dans André Magord (dir.), L'Acadie plurielle - Dynamiques identitaires collectives et développement au sein des réalités acadiennes, avec la coll. de Maurice Basque et Amélie Giroux, Moncton / Poitiers, Université de Moncton, Centre d'études acadiennes / Université de Poitiers, Institut d'études acadiennes et québécoises, 2003, p. 409-422.

10. Parmi ceux-ci, les articles de David Bélanger abordent certains enjeux entourant la critique artistique, puisqu'il arrive qu'elle fasse elle-même - son rôle, ses choix, ses éventuels conflits d'intérêts, etc. - l'objet de querelles littéraires, ou que ces dernières y transparaissent. Voir : David Bélanger, "La réception de la littérature en Acadie : sur quelques débats autour de la critique dans L'Évangéline et dans L'Acadie Nouvelle ", Voix plurielles, vol. 9, $\mathrm{n}^{\circ}$ 2, 2012, p. 149-163; Benoit Doyon-Gosselin et David Bélanger, « D'une querelle l'autre : l'autonomisation de la littérature acadienne », Romanica Silesiana, $\mathrm{n}^{\circ}$ 7, 2012, p. 187-198; David Bélanger, "Controverses et concurrences : l'inquiétude critique dans L'Acadie Nouvelle (1991-1993) », dans Benoit Doyon-Gosselin, David Bélanger et Cassie Bérard (dir.), Les institutions littéraires en question dans la Franco-Amérique, Québec, Presses de l’Université Laval, coll. «Culture française d'Amérique », 2014, p. 359-378. Voir aussi : Sébastien Hogue, «Des racines et des ailes : le milieu théâtral acadien sur la corde raide », dans Benoit Doyon-Gosselin, David Bélanger et Cassie Bérard (dir.), Les institutions littéraires en question dans la Franco-Amérique, Québec, Presses de l’Université Laval, coll. « Culture française d’Amérique », 2014, p. 335-357.

11. Voir Robert Viau, "Querelles, trahisons et gloire : les chemins du Goncourt », dans Acadie multipiste, t. 1, Moncton, Perce-Neige, 2015, p. 47-86 et Benoit Doyon-Gosselin, "Les prix et la méprise : primes et déprimes de l'institution littéraire acadienne ", dans Benoit Doyon-Gosselin, David Bélanger et Cassie Bérard (dir.), Les institutions littéraires en question dans la Franco-Amérique, 
mais il n'existe pas d'étude consacrée à la pratique de la critique artistique en Acadie $^{12}$, sinon des réflexions courtes de David Lonergan ${ }^{13}$. Le présent article vise à remédier à ce vide, d'abord en documentant ce qu'on désigne souvent comme une "crise », mais qui perdure pourtant en Acadie depuis une dizaine d'années, et ensuite, en établissant le profil de la critique en milieu minoritaire : on observera ainsi que certains enjeux récurrents viennent compliquer la mise en place d'une tradition durable. Mais avant d'y parvenir, tentons de définir la critique en la replaçant dans son contexte institutionnel, c'est-à-dire dans la chaîne de production culturelle.

\section{Formes et fonctions de la critique artistique}

Dans la chaîne de production culturelle, l'activité critique occupe une position singulière, puisqu'elle amorce, comme l'explique Joseph Jurt dans son étude de la réception littéraire, un second cycle de communication $^{14}$. Le critique est d'abord un public de l'art; de récepteur, il devient ensuite émetteur et engendre sa propre réception en interpellant de nouveaux publics (ceux de la tribune où il écrit). C'est à partir de cette caractéristique que l'on peut comprendre le mot de Roland Barthes pour qui "le critique, pas plus que l'écrivain, n'a jamais le dernier mot »" L'étrange discussion ainsi provoquée est par nature axiomatique, selon la définition générale de la critique que propose Robert Dion : "La critique désigne l'art d'appliquer des critères, donc de porter un jugement. [...] Dans sa plus grande généralité, [le mot "critique »] indique des pratiques qui ont accompagné la vie des lettres depuis l'Antiquité : l'évaluation et l'interprétation des œuvres. ${ }^{16}$ La boutade veut même que le premier artiste de la civilisation humaine, en dessinant des figures dans les grottes ornées de la préhistoire, ait du même coup inventé l'activité critique.

Québec, Presses de l’Université Laval, coll. « Culture française d’Amérique », 2014, p. 137-158.

12. Boudreau déplore aussi l'absence de recherches sociologiques sur le lectorat de la littérature acadienne. Voir Raoul Boudreau, « L’institutionnalisation inachevée de la littérature acadienne », op. cit., p. 157.

13. Voir David Lonergan, "La critique dans un petit milieu telle que vécue par un praticien », dans Tintamarre : chroniques de littérature dans l'Acadie d'aujourd'hui, Sudbury, Prise de parole, coll. «Agora », 2008, p. 315-322.

14. Voir Joseph Jurt, La réception de la littérature par la critique journalistique - Lectures de Bernanos 1926-1936, Paris, J.-M. Place, 1980, p. 37.

15. Roland Barthes, Essais critiques, Paris, Seuil, coll. «Tel quel », 1964, p. 9. L'italique est de l'auteur.

16. Robert Dion, "Critique littéraire », dans Paul Aron, Denis Saint-Jacques et Alain Viala (dir.), Le dictionnaire du littéraire, Paris, Presses universitaires de France, 2004 [2002], p. 134. 
L'examen de la critique artistique comme production de discours est complexifié du fait qu'elle n'est pas unique, qu'elle prend diverses formes. En effet, on distingue traditionnellement une critique universitaire, une critique d'artiste et une critique journalistique, selon la tripartition d'Albert Thibaudet (nous y reviendrons) ${ }^{17}$, qui a concouru, grâce à son ouvrage Réflexions sur la critique, à donner ses lettres de noblesse au genre. Pour mieux situer l'activité critique dans l'institution culturelle, il s'agit de voir comment elle navigue entre chacun des domaines qu'elle convoque l'université, l'art et le journalisme. La porosité, ou la perméabilité, entre ces milieux fait de la critique artistique un discours par nature hybride, ou « singulier pluriel $»^{18}$, selon la formule de Laurent Jenny, qui considère que la critique artistique ne réalise son plein potentiel qu'en rassemblant l'expertise des trois formes de la critique :

Pour que la Critique existe, il faut qu'elle soit trois en une, c'est-à-dire que ces trois critiques ne soient pas des spécialités rigoureusement étanches, mais qu'il y ait entre elles la possibilité de nombreux échanges et circulations qui font la vie même de la Critique. ${ }^{19}$

Exacerbée dans les petites institutions culturelles, cette porosité excentre cependant la responsabilité de la production de la critique et en fait une véritable patate chaude.

La critique artistique relève-t-elle de la responsabilité des universitaires? Certes, on parle de critique pour désigner un discours théorique sur la littérature ou une méthode d'analyse des œuvres littéraires - et on les nomme parfois ainsi : critique génétique, « nouvelle critique ", sociocritique, etc. Mais la recherche universitaire est une réception différée, non immédiate de la production artistique, et ne porte pas nécessairement sur l'actualité artistique; les chercheurs sont même parfois réticents à l'idée de travailler sur des œuvres récentes, bien que ce soit moins le cas en milieu minoritaire, et de moins en moins le cas ailleurs ${ }^{20}$. De plus, même lorsqu'il se penche sur la production

17. Voir Albert Thibaudet, Réflexions sur la critique, $2^{\mathrm{e}}$ édition, Paris, Gallimard, 1939, p. $125-136$.

18. Laurent Jenny, «État critique », Les Temps Modernes, n 672, 2013, p. 65.

19. Ibid., p. 64 .

20. Même en France, Thierry Guichard observe que «la critique universitaire [...], depuis quelques années, abandonne de plus en plus les dépouilles littéraires pour se tourner vers "l'extrême contemporain" " (Thierry Guichard, "Le fait littéraire ", Les Temps Modernes, $n^{\circ}$ 672, 2013, p. 200). Dans le même numéro, Laurent Jenny fait pourtant le constat inverse : "les études littéraires [...] renoncent spontanément à se reconnaître dans le rapport vivant qu'elles entretenaient à l'art littéraire » 
contemporaine, le travail universitaire n'est pas nécessairement de nature axiomatique. Cependant, nombre des gens qui font de la critique immédiate (dans les journaux ou les revues culturelles) sont soit des professeurs soit des étudiants. L'ombre du milieu universitaire plane assurément sur la critique artistique, d'autant plus que les entreprises de vulgarisation sont de plus en plus valorisées dans certains milieux de recherche.

La critique artistique relève-t-elle de la responsabilité des artistes? En parlant de critique d'artiste, Thibaudet renvoie surtout à la tradition, très présente dans l'histoire de la littérature française, mais de plus en plus rare en période contemporaine, d'auteurs qui proposent des lectures, parfois très judicieuses, d'autres auteurs ${ }^{21}$. L'intérêt de l'exercice se situe cependant presque autant dans le développement ou la confirmation de leur propre vision esthétique. Mais il existe une seconde façon d'envisager la relation entre la critique et le monde des arts. Dans la perspective où la critique artistique a la double fonction de récepteur-émetteur dans la communication entre le créateur et le public, le critique doit-il être considéré comme un écrivain? la critique artistique comme un genre littéraire? Pour Roland Barthes, on ne saurait l'envisager autrement ${ }^{22}$. Participant de la littérature d'idées d'une façon similaire à l'essai, dont l'apport est à la fois scientifique et esthétique, la critique aurait une double intention interprétative, mettant à profit la polysémie du mot interpréter, comme l'explique la sociologue de l'art Nathalie Heinich :

Aussi faut-il entendre " interprétation » au double sens du terme : « interpréter » une œuvre d'art contemporaine n'est pas seulement, pour un critique, l'expliquer, c'est aussi l'exécuter, comme on le dit d'une œuvre musicale; c'est la faire exister par sa mise en circulation dans le monde, grâce à la médiation d'un travail discursif - entre mise en intrigue et mise en énigme - qui doit beaucoup à la qualité de l'interprète. ${ }^{23}$

Cependant, ce type de critique fait exception. Même lorsqu'un critique a le souci de développer un style et une voix originaux, la critique artistique, en particulier la réception immédiate, s'élève rarement au rang de proposition esthétique ${ }^{24}$.

(Laurent Jenny, op. cit., p. 66).

21. Pensons entre autres à Hugo lisant Shakespeare, Sartre revenant sur Baudelaire et Flaubert ou, plus récemment, les essais de Milan Kundera.

22. Voir Roland Barthes, op. cit., p. 9.

23. Nathalie Heinich, Le triple jeu de l'art contemporain, Paris, Minuit, coll. «Paradoxe », 1998, p. 324.

24. Nonobstant la proposition de Chiasson citée en début d'article, selon laquelle 
Enfin, la critique artistique relève-t-elle de la responsabilité des journalistes, ou plus largement des médias? D’abord, il faut distinguer la critique artistique (qui interprète et porte un jugement sur l'œuvre en l'inscrivant dans un plus grand contexte) du journalisme culturel (qui est un discours essentiellement promotionnel, constitué d'annonces d'événements, d'entrevues avec les artistes ou de comptes rendus). Cette distinction n'est pas toujours évidente à faire (ni pour le milieu journalistique ni pour le grand public) dans le contexte actuel de réduction croissante de l'espace médiatique accordé aux arts ni dans le contexte minoritaire, où les gens portent souvent plusieurs chapeaux, écrivant par exemple à la fois des prépapiers et des critiques. Mais il y a une irréductibilité de la critique artistique, puisqu'elle est le seul discours - et c'est là toute son importance - à porter spécifiquement et exclusivement sur l'œuvre d'art, comme le rappelle la critique américaine Gail Pool : «The traditional review is the only kind of "coverage" that focuses exclusively on the text and that is-or at least aims to be-written by a disinterested and qualified critic. $»^{25}$

Pour comprendre le statut particulier de la critique artistique dans l'univers médiatique, il faut remonter aux origines de l'émergence d'un discours critique dans les journaux. Ce n'est que rétrospectivement qu'on a appelé «critique journalistique » l'une des trois critiques qui intéressent Thibaudet, puisque lui-même parle de "critique parlée ", et la définit de façon minimale comme « la critique spontanée, la critique faite par le public

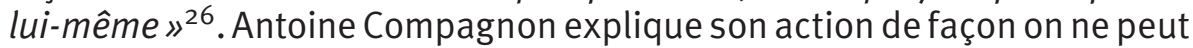
plus concrète : " [l]a critique commence quand on parle autour de la table du dernier film sorti dans les salles ou des romans de la rentrée ${ }^{27}$. Pour Laurent Jenny, cette critique ne serait pas si démocratique que l'entend Thibaudet, puisqu'elle est le résultat de la logique institutionnelle de la vie littéraire des $\mathrm{XVII}^{\mathrm{e}}$ et $\mathrm{xVIII}{ }^{\mathrm{e}}$ siècles : " dès l'origine, elle émane en réalité d'un groupe social défini : elle naît de la vie de société et de conversation, c'est-à-dire des salons ${ }^{28}$. Elle est devenue réellement " journalistique » - et, par la même occasion, plus prescriptive, selon Jenny ${ }^{29}$ - lors de sa

les créateurs acadiens doivent être les premiers spectateurs et critiques de l'art acadien, la critique d'artiste est à peu près inexistante en Acadie, si ce n'est dans les préfaces à des rééditions.

25. Gail Pool, Faint Praise - The Plight of Book Reviewing in America, Columbia, University of Missouri Press, 2007, p. 120.

26. Albert Thibaudet, op. cit., p. 126.

27. Antoine Compagnon, "Quand les écrivains ne s'aimaient pas », Les Temps Modernes, $n^{\circ} 672,2013$, p. 8.

28. Laurent Jenny, op. cit., p. 63.

29. Ibid. 
professionnalisation et de son déplacement dans les médias, au cours du $\mathrm{XIX} \mathrm{x}^{\mathrm{e}}$ siècle.

Pour Compagnon, l'ère numérique permet à la « critique parlée » de revenir en force :

En effet, nous sommes entrés dans un nouvel âge de la conversation : la critique immédiate a lieu sur Internet, dans les blogs, les commentaires des librairies en ligne. Et c'est très bien. Alors que les jugements des critiques patentés étaient à prendre ou à laisser, l'autorité des chroniqueurs numériques est instantanément battue en brèche par le petit peuple des anonymes. ${ }^{30}$

La critique journalistique a maintenant à se définir en fonction de ce qui s'écrit sur la toile, mais ce ne sont pas tous les commentateurs qui envisagent le phénomène avec l'enthousiasme de Compagnon. À côté de ces jugements certes démocratiques, la critique artistique, tout en demeurant une réception immédiate, est une opinion " professionnelle », c'est-à-dire qui s'octroie une certaine responsabilité (en plus d'une autorité), en passant du domaine privé au domaine public, et qui conserve son importance d'autant plus que les opinions des simples amateurs sont de plus en plus nombreuses et diffusées. Pour Gail Pool, "[o]pinion is needed, and as the surge of opinions on the Web makes clear, it is very much in demand. Whatever the format and whatever the technology, we need book reviewing that plays "a filtering role" - and plays it well ${ }^{31}$.

Replacer ainsi la critique comme maillon essentiel dans la grande chaîne culturelle, quoiqu'à cheval entre ces différentes sphères que sont l'université, le milieu artistique et le journalisme, permet de dégager certaines de ses fonctions. On peut d'emblée faire la distinction entre les rôles de la critique associés à ses publics immédiats (les instances de production et de diffusion, les artistes, le grand public) et son rôle projeté dans l'avenir, pour un public différé, c'est-à-dire par rapport à la mémorialisation de l'art. Du côté des instances de production (comme les maisons d'édition, les agents d'artistes ou les galeries d'art), la critique permet de se construire un gage de qualité, qui peut servir à obtenir des subventions. Pour ce qui est des artistes, on ne peut négliger l'importance des réactions aux œuvres, dans un domaine où, l'originalité étant la valeur primordiale, les artistes ne peuvent se tourner vers un discours passé ou conventionnel pour confirmer leur travail. La critique est aussi un intermédiaire entre l'art et son public. Pour reprendre la

30. Antoine Compagnon, op. cit., p. 9

31. Gail Pool, op. cit., p. 14 . 
réflexion de Catherine Voyer-Léger, la " critique idéale " situe l'œuvre dans ses multiples contextes : de la discipline artistique, des conventions esthétiques, de la société ou de la communauté, et enfin du travail précédent de l'artiste ${ }^{32}$. Enfin, la mémorialisation de l'art est une fonction particulièrement importante de la critique artistique, et particulièrement en milieu minoritaire, où bien souvent la critique est le seul retour que connaîtra une œuvre.

\section{La « crise » de la critique artistique en Acadie}

La principale conséquence de la disparition de «Tintamarre » dans le journal L'Acadie Nouvelle en 2013 est précisément de rendre plus difficile la mise en mémoire de l'art acadien. Il est évident que les meilleures conditions de mémorialisation de l'art sont réunies lorsqu'un espace médiatique de critique artistique devient une référence, c'est-à-dire un discours continu, plutôt qu'une prise de parole circonstancielle ${ }^{33}$, et qui puisse éventuellement être relayé par la recherche et l'enseignement universitaires. Sans cette mise en valeur du présent qui devient mémoire du passé, il ya, en définitive, coupure de l'art avec son public contemporain et futur.

D’où la commotion quand, le 25 septembre 2013, David Lonergan annonçait sur sa page Facebook personnelle que L'Acadie Nouvelle avait éliminé son espace de critique artistique, la chronique "Tintamarre », en raison d'un lectorat jugé insuffisant. Selon un sondage interne, elle était lue par 11,5 p. 100 des lecteurs réguliers du journal et 25,8 p. 100 des lecteurs occasionnels ${ }^{34}$. Or ces chiffres sont comparables aux résultats de sondages effectués ailleurs : une enquête menée par Berend $H$. Fedderson en Allemagne révèle que "les comptes rendus des livres sont lus par moins de 10 p. 100 des lecteurs d'un journal (si le livre est d'un intérêt local éminent, le taux de lecture peut être plus élevé, mais il ne dépasse jamais 20 p. 100) $»^{35}$. Force est de constater que les attentes de L'Acadie Nouvelle étaient démesurées ${ }^{36}$.

32. Voir Catherine Voyer-Léger, Métier critique, Montréal, Septentrion, 2014, p. 76 et 77.

33. De la même façon, la publication en ouvrage des critiques littéraires de David Lonergan leur assure une meilleure postérité, en leur donnant une nouvelle envergure et en les rendant plus accessibles. Voir David Lonergan, Tintamarre, op. cit.

34. Courriel de Jean Saint-Cyr à David Lonergan, 26 août 2013.

35. Enquête citée dans Joseph Jurt, op. cit., p. 41.

36. Il convient de préciser que L'Acadie Nouvelle n'évaluait pas le rendement de «Tintamarre » en fonction des espaces à contenu culturel ou de critique artistique dans d'autres médias, mais bien à l'intérieur même du journal, où la chronique de Lonergan aurait obtenu «le plus bas score de toutes les chroniques » (courriel 
Ce n'était toutefois pas la première fois qu'on interrompait « Tintamarre » ni que l'existence d'un espace médiatique de critique artistique était matière à discussion publique en Acadie. En fait, on peut parler à juste titre d'une normalisation de la notion de «crise » en ce qui concerne la critique artistique acadienne, puisque sa situation précaire remonte à bien avant l'établissement ou la mise au rancart de "Tintamarre ». Au tournant des années 1990, principalement sous la plume du poète Martin Pitre, L'Acadie Nouvelle revenait régulièrement sur les nouveautés littéraires acadiennes. Un espace désigné « Les livres au Chat Pitre » figurait même souvent dans les pages culturelles du journal, s'approchant d'une critique artistique et donnant une bonne place à des collaborations spéciales ${ }^{37}$, mais ne portant pas exclusivement sur des œuvres acadiennes ${ }^{38}$.

Mais "Les livres au Chat Pitre » ne paraissait plus depuis deux ans lorsque David Lonergan, homme de théâtre originaire de la Gaspésie, emménage à Moncton en 1994 pour y entreprendre des études supérieures. C'est au moment où il commence, à l'automne de cette année-là, à écrire régulièrement pour le journal qu'on peut réellement dire qu'un espace médiatique de critique artistique porte délibérément, explicitement et exclusivement sur les arts acadiens : "En accord avec la direction du journal, signale-t-il dans l'un de ses premiers textes, j'ai décidé de spécialiser cette chronique à ce qui se passe et s'écrit ici. „39

Entre 2001 et 2003, après plusieurs années d'écriture hebdomadaire ou même bihebdomadaire, Lonergan (devenu professeur de journalisme à l'Université de Moncton) diminue la fréquence de ses critiques à une par mois. Pendant deux ans, entre 2004 et 2006, il recrute deux étudiants de cycle supérieur en littérature, Pénélope Cormier puis Clint Bruce, pour l'aider à tenir une chronique hebdomadaire. Cependant, le journal met un terme à "Tintamarre » en 2006, face à ce qu'il perçoit comme une instabilité de l'espace médiatique. À la suite de la réaction des milieux artistique et universitaire $4^{\circ}$, la direction du journal publie une mise au point, se défendant notamment d'avoir retranché la chronique faute de

de Jean Saint-Cyr à David Lonergan, 26 août 2013). Cela signifie néanmoins que la spécificité de la critique artistique parmi les types de discours journalistique n'a pas été prise en compte comme facteur dans la décision de la direction du journal.

37. Outre Martin Pitre, le collaborateur qui aborde le plus souvent des œuvres acadiennes est Raoul Boudreau, professeur de littérature à l'Université de Moncton.

38. On y présente le dictionnaire Larousse dans une chronique d'avril 1991!

39. David Lonergan, "Critique littéraire : danger », L’Acadie Nouvelle, 6 janvier 1995 , p. 30.

40. On compte au moins deux lettres à l'opinion du lecteur, de Benoit Doyon-Gosselin, alors étudiant de cycle supérieur en littérature, et de Zénon Chiasson, anciennement professeur de littérature. 
lecteurs. L'Acadie Nouvelle se dit même prête à rétablir "Tintamarre ", à la condition que Lonergan s'engage à écrire toutes les semaines, pour rétablir une « voix » propre à la chronique :

\begin{abstract}
La direction du journal insiste sur l'importance que revêt la régularité de la parution des chroniques, quel que soit le domaine visé par la chronique. [...] Reconnaissant qu’il est crucial pour une chronique de paraître régulièrement, $M$. Lonergan a accepté de maintenir une chronique hebdomadaire à partir du début février. ${ }^{41}$
\end{abstract}

Le retour de Lonergan marquait la fin de la crise, qu'il avait d'ailleurs lui-même anticipée en 2005 dans un numéro spécial sur la littérature franco-canadienne de la revue d'actualité artistique Liaison, qui était alors consacrée à l'Ontario français : "La critique littéraire n'a guère d'espace en Acadie. [...] Ceux qui créent se limitent à la création, les universitaires ont assez à faire avec les revues et les colloques spécialisés. Il y a peu ou pas de vocation sinon des vocations temporaires. ${ }^{42}$ Le constat ne pourrait être plus clair. Ce n'est pas faute d'espace médiatique que la critique est quasi inexistante en Acadie, mais faute de volontaires prêts à faire le pont entre un discours spécialisé (universitaire ou artistique) et un discours destiné au grand public ${ }^{43}$. La meilleure preuve que le problème n'est pas dû au manque d'espaces médiatiques, c'est qu'un an après la parution de ce numéro spécial, la revue trimestrielle Liaison élargissait son mandat à l'ensemble des arts franco-canadiens.

En Acadie cependant, la situation s'est inversée depuis ce premier arrêt de la chronique. Revenons au 25 septembre 2013 sur Facebook, où les réactions à la coupure de "Tintamarre " sont aussi virulentes et nombreuses qu'en 2006. Le brouhaha débouche sur l'espace public et dure plusieurs mois : en septembre, la télévision de Radio-Canada fait un reportage sur les réactions d'agents du milieu artistique; en février, l'Association acadienne des journalistes (AAJ) organise une table ronde sur le sujet ${ }^{44}$; en mars, le sociologue Mathieu Wade cherche à trouver des

41. [s.a.], " La critique culturelle à L'Acadie Nouvelle - Mise au point ", L'Acadie Nouvelle, cahier "L'accent acadien », 4 novembre 2006, p. 2.

42. David Lonergan, "La fragile édition acadienne ", Liaison, n 129, 2005, p. 51.

43. La capacité qu'a Lonergan de cibler le public du journal est reconnue comme l'une des forces de ses critiques : "Les textes de Lonergan, à travers L'Acadie Nouvelle, ont donc permis à plusieurs auteurs acadiens de sortir du giron universitaire et d'entrer dans les foyers du Nouveau-Brunswick, où ils ont pu rejoindre un lectorat non spécialiste. » (Jimmy Thibeault, "David Lonergan, Tintamarre », @nalyses, vol. $5, \mathrm{n}^{\circ} 1,2010$, p. 219)

44. Tenue le 24 février 2014 à l'Université de Moncton, la table ronde réunissait David 
raisons à l'absence de critique artistique en Acadie dans un article sur le webzine acadien Astheure 45 .

Ce qui a changé, depuis 2006, ce ne sont pas les réactions, mais la raison donnée par la direction de L'Acadie Nouvelle pour l'abandon de la chronique - le faible lectorat - et son absence remarquée du débat public à la suite de sa décision. Une lettre d'opinion d'Herménégilde Chiasson, critiquant la décision du journal, n'a pas été publiée; le rédacteur en chef s'est contenté de lui répondre en privé. En 2013, contrairement à 2006, il y a aussi des volontaires prêts à prendre la relève de Lonergan. À l'automne 2014, Benoit Doyon-Gosselin, professeur de littérature à l'Université de Moncton et titulaire de la Chaire de recherche du Canada en études acadiennes et milieux minoritaires, a proposé à la direction du journal d'écrire une critique littéraire - gratuitement -, mais elle a refusé ${ }^{46}$. Ce qui différencie la crise de 2013 de la crise de 2006, c'est donc l'absence catégorique de volonté, de la part du quotidien, de maintenir un espace de critique artistique dans ses pages. Bien sûr, le journal n'a pas créé le problème du développement durable d'une critique artistique en Acadie; on peut même dire que, pendant près de vingt ans, il a contribué, par son maintien d'un espace médiatique réservé à la critique artistique acadienne, à offrir une sorte de seuil minimal qui rendait moins dramatique la situation de la critique artistique. Cela dit, si la « crise » de la critique artistique en Acadie est un état permanent, il demeure qu'elle est certainement plus criante depuis la disparition de «Tintamarre ».

\section{Au-delà de la crise : enjeux de la critique en milieu minoritaire}

Depuis une dizaine d'années, les aléas spécifiques de "Tintamarre » ont suscité un grand nombre de réactions au sein des milieux universitaire, artistique et journalistique. Les enjeux soulevés, qui sont toujours les mêmes, méritent qu'on s'y attarde, afin d'établir le profil de la critique culturelle en milieu minoritaire. Sans négliger de reconnaître ses réels défis, nous tenterons de débusquer certaines idées reçues et d'en relativiser d'autres.

Lonergan, la journaliste culturelle et artiste au théâtre Katherine Kilfoil, le directeur général de la Fédération culturelle acadienne de la Nouvelle-Écosse Martin Théberge et l'auteur-compositeur-interprète Serge Brideau, avec le président de l'AAJ, Pascal Raiche-Nogue, comme animateur.

45. Voir Mathieu Wade, "Blind spot de la littérature acadienne », Astheure, 31 mars 2014; sur Internet : http://astheure.com/2014/03/31/blind-spot-de-la-litteratur e-acadienne-mathieu-wade/ (page consultée le 28 décembre 2015).

46. Courriels entre Benoit Doyon-Gosselin et Francis Sonier, éditeur-directeur général d'Acadie média, du $1^{\text {er }}$ au 4 septembre 2014 . 
L'un des enjeux qui reviennent le plus souvent concerne le nombre insuffisant de critiques, dans le sens de personnes aptes à commenter et à juger les œuvres. En 2005, David Lonergan écrivait que le principal élément expliquant l'absence de critiques dans les médias est "l'absence de personnes volontaires pour exercer [c]e délicat métier "47. Arash Mohtashami-Maali, alors directeur et rédacteur en chef de Liaison, est du même avis. La constitution d'une équipe de collaborateurs de taille suffisante pour produire une revue artistique en milieu minoritaire représente un défi de taille : "[C]omment trouver un collaborateur pour parler d'une cuvre en s'assurant qu'il est spécialiste du domaine, qu'il n'est pas en conflit d'intérêts, qu'il a le temps de rédiger un article[?] „48

Le problème se pose moins pour la littérature que les arts visuels, la danse ou la musique (surtout instrumentale). La critique de ces disciplines artistiques suppose un travail additionnel : la transposition médiatique, c'est-à-dire l'adoption d'un code (le langage écrit) autre que celui employé pour produire l'œuvre. Au sujet de la critique cinématographique, Michel Larouche et Serge Cardinal font falloir que l'écriture est employée pour " traduire le propre d'une narration cinématographique qui associe images et sons et procède par ellipses, sous-entendus, associations d'idées »49. Or les diplômés en beaux-arts ne sont pas forcément formés pour accomplir ce type de travail, qui nécessite des aptitudes en écriture et en analyse, puisque leur parcours universitaire est principalement axé sur la pratique. En revanche, les diplômés en littérature n'ont pas nécessairement les connaissances pour décrire un tableau, un spectacle de danse ou un album de musique en employant le vocabulaire adéquat ni pour situer une telle œuvre dans le contexte de sa discipline.

Pourtant, le nombre restreint de collaborateurs fait en sorte qu'un critique comme Lonergan sera sans doute appelé, au cours de sa carrière, à produire des textes sur des disciplines artistiques avec lesquelles il est moins à l'aise. "Écrire sur les arts dans un petit milieu, nous dit-il, demande un éclectisme amusé et une bonne dose de modestie puisque l'on parle bien souvent à partir de son ignorance. "50 Pour Gail Pool, la disparition des journalistes culturels spécialisés (qui se consacrent à une seule discipline) au profit des chroniqueurs généralistes, disparition qui

47. David Lonergan, «La fragile édition acadienne », op. cit., p. 51.

48. Arash Mohtashami-Maali, "La revue Liaison, entre le marteau et l'enclume ", Liaison, $\mathrm{n}^{\circ} 142$, 2008-2009, p. 13.

49. Michel Larouche et Serge Cardinal, "L'écriture de la critique », Cinémas : revue d'études cinématographiques / Cinemas : Journal of Film Studies, vol. 6, n²-3, 1996, p. 131.

50. David Lonergan, «La critique dans un petit milieu telle que vécue par un praticien », op. cit., p. 319. 
est allée en augmentant tout au long du xxe siècle, ne pose pas de véritable problème ${ }^{51}$. Les spécialistes, souvent des chercheurs universitaires, peinent à vulgariser leurs idées et à respecter des échéances serrées ${ }^{52}$. Selon elle, les généralistes peuvent accomplir un bon travail, à condition de compléter les lectures et la recherche qui s'imposent pour aborder une $œ u v r{ }^{53}$. Encore faut-il que les critiques, habituellement des pigistes, en aient les moyens : leur faible revenu (quand il ne s'agit pas d'un travail bénévole) fait en sorte qu'ils peuvent choisir de limiter le temps consacré à l'écriture d'une critique ou " préférer les entrevues et les prépapiers, souvent moins exigeants, moins risqués, moins pressants et plus longs, donc plus payants ${ }^{54}$. En général, ce sont les conditions de travail imposées par le milieu plutôt que le profil des critiques qui entravent leur pratique.

Le travail de critique ne requiert pas de formation officielle, même dans les milieux les mieux nantis (lire : majoritaires). Pool souligne que " qualification plays an uncertain role in hiring: the reviewers who are published may be qualified to critically assess books and explain their assessments, or they may not ${ }^{55}$. La critique, qui œuvre aux États-Unis, ne se souvient pas d'avoir fourni des renseignements sur sa formation universitaire ni même une copie de son CV pour être embauchée ${ }^{56}$. Voyer-Léger affirme que «ce n'est pas le diplôme qui fait le critique »57, car, malgré une prépondérance de diplômés en journalisme, les "parcours menant à ce métier sont multiples ${ }^{58}$. Dans la francophonie canadienne, de récentes initiatives visent néanmoins à aiguillonner les personnes intéressées dans la bonne direction. Le projet "L'art visuel s'écrit » de l'Association des groupes en arts visuels francophones (AGAVF) cherche notamment à susciter un intérêt pour l'écriture des arts actuels en rendant disponibles des documents de réflexion, ainsi qu'en donnant des formations sur les enjeux d'une telle pratique à travers les communautés francophones minoritaires du pays, dont l'Acadie. 59

Lorsque les critiques ont les compétences et la volonté nécessaires pour en faire, ils sont souvent en conflit d'intérêts. La petitesse du milieu

51. Au contraire, Voyer-Léger voit d'un mauvais d'œil la disparition des spécialistes. Voir Catherine Voyer-Léger, Métier critique, op. cit., p. 36-40.

52. Voir Gail Pool, op. cit., p. 54 et 55.

53. Ibid., p. 56.

54. Catherine Voyer-Léger, op. cit., p. 36.

55. Gail Pool, op. cit., p. 39.

56. Ibid., p. 40.

57. Catherine Voyer-Léger, op. cit., p. 34.

58. Ibid. p. 33.

59. Voir le site Web de « L'art visuel s'écrit »: http://www.agavf.ca/dossiers/ artvisuelecrit.html 
artistique constitue l'enjeu le plus souvent évoqué quant à la critique, en contexte minoritaire comme en contexte majoritaire : "Même le milieu littéraire français se plaint d'une trop grande promiscuité! "60 Le manque d'effectifs - tout comme le travail à la pige du critique - fait en sorte que la plupart des acteurs culturels cumulent plusieurs rôles. Il n'est pas rare pour un critique d'être à la fois auteur publié et professeur d'université; de travailler pour une institution tout en étant membre du conseil d'administration d'une autre; et ainsi de suite. C'est sans parler des réseaux personnels des critiques, qui ont souvent côtoyé les artistes de leur génération au cours de leurs études. Les autres, ils risquent tôt ou tard de les croiser lors des nombreux lancements, assemblées annuelles, regroupements associatifs et cocktails auxquels leurs fonctions dans le milieu culturel les convoquent. Si ce ne sont pas les artistes eux-mêmes qu'ils connaissent, alors ce sont les autres membres de la chaîne de production artistique. Évidemment, cette exiguïté est exacerbée en milieu minoritaire.

Or l'un des dangers de la proximité, c'est justement que "l'amour rend aveugle ${ }^{61}$. Certains retours d'ascenseurs, même inconscients, sont susceptibles de se produire. En lisant une critique rédigée par un écrivain sur le travail d'un collègue, il faut, selon Jules Tessier, " savoir lire entre les lignes, être à l'affût du moindre indice qui permette de détecter les réserves, les réticences, car il y a de fortes chances que les aspects négatifs aient été édulcorés, sinon carrément gommés ${ }^{62}$. Son analyse de six critiques réciproques entre Andrée Lacelle et trois écrivaines franco-ontariennes - dont la moitié, écrite par Lacelle, porte sur l'œuvre de ces écrivaines et l'autre moitié, écrite par ces écrivaines, porte sur l'œuvre de Lacelle - le montre bien: " on cherche en vain un commentaire négatif; on trouve tout au plus des degrés variables d'enthousiasme " $" 33$.

Certaines solutions permettent néanmoins d'atténuer les conflits d'intérêts et d'instaurer le minimum de distance nécessaire pour faire de la critique, même en contexte d'exiguïté. Il est plus facile, par exemple, pour un critique d'effectuer son travail s'il est d'abord connu en tant que tel dans son milieu : "ce qui légitime la parole du critique, c'est à la fois la scène où il agit et le capital symbolique accumulé au long de sa trajectoire personnelle ${ }^{64}$. Le désir d'accumuler un tel capital peut entraîner chez

60. Catherine Voyer-Léger, «Réflexions sur la relation critique », Liaison, $n^{\circ}$ 167, 2015 , p. 6.

61. Catherine Voyer-Léger, Métier critique, op. cit., p. 69.

62. Jules Tessier, "Andrée Lacelle et la critique », Francophonie d'Amérique, $n^{\circ} 11$, 2001 , p. 95.

63. Ibid., p. 96.

64. Dominique Bertelli, «La réception du fait littéraire par la critique journalistique », 
le critique le refus d'assumer d'autres fonctions dans le milieu artistique - choix déchirant si celles-ci sont rémunérées, puisque le travail à la pige ne paie pas suffisamment pour en vivre. Le critique qui est déjà impliqué autrement dans sa communauté artistique gagnerait probablement à évaluer les œuvres d'une autre discipline ou d'une autre région géographique que la sienne, à condition d'avoir la légitimité nécessaire pour le faire. En général, il importe que le critique réfléchisse au lieu à partir duquel il écrit, notamment à ses subjectivités. Comme les conflits d'intérêts sont imperceptibles pour les lecteurs de la critique (hormis ceux qui sont également impliqués dans le milieu), un travail de transparence est également nécessaire.

L'exiguïté du milieu fait en sorte qu'on a souvent l'impression qu'en situation minoritaire, le critique n'est pas vraiment critique : il ne peut jamais exprimer le fond de sa pensée, car les artistes réagissent mal aux commentaires défavorables. Il a certes été difficile d'établir une tradition de critique artistique au Canada francophone. Lorsqu'il s'est joint au comité de Liaison au début des années 1980, François Paré se souvient que le directeur de la revue lui a recommandé d'" éviter un comportement critique, un ton de voix, une gestuelle qui seraient immédiatement perçus par [s]es collègues comme de l'arrogance "65. Pour sa part, Arash Mohtashami-Maali, à la barre de Liaison au cours des années 2000, avouait devoir "passer un certain temps à répondre aux mécontents "66 après la parution de chaque numéro. Mais ces exceptions ne justifient pas la règle. De l'avis de Lonergan, "[d]ans la presque totalité des cas, les réactions [à la critique négative] sont saines : on peut être en désaccord, mais on ne le prend pas "personnel" "67.

Les appréhensions des critiques quant à la réaction des artistes ne devraient pas les inciter à signer des textes trop élogieux. Car, comme se le demande Voyer-Léger : "À quoi servirait l'espace critique si la potentialité d'une critique négative n'existait pas? "68 D'un autre côté, il n'est pas particulièrement constructif de recenser une œuvre si ce n'est que pour l'anéantir : "une telle attitude finira aussi par dessécher le milieu ${ }^{69}$. La remarque s'impose à plus forte raison pour le milieu minoritaire, où la majorité des productions artistiques font rarement

Questions de communication, n 8, 2005, p. 173.

65. François Paré, Les littératures de l'exiguïté, Ottawa, Le Nordir, coll. «Bibliothèque canadienne-française », 2001 [1992], p. 39.

66. Arash Mohtashami-Maali, op. cit., p. 12.

67. David Lonergan, «La critique dans un petit milieu telle que vécue par un praticien », op. cit., p. 319.

68. Catherine Voyer-Léger, «Réflexions sur la relation critique », op. cit., p. 7.

69. Ibid., p. 7. 
l'objet de plus d'une critique. Le plaidoyer de certains chercheurs pour une critique universitaire adaptée aux milieux minoritaires, critique qui tiendrait compte du contexte d'énonciation, vaut tout autant pour la critique journalistique ${ }^{70}$. Un tel assouplissement dans la façon d'aborder une œuvre n'implique pas de garder pour soi ses réserves, mais de les communiquer en faisant preuve de doigté, pour demeurer juste et nuancé. Pour reprendre la formule de Mathieu Charlebois, "[l]e critique n'a pas à être gentil [envers les artistes] : il a le devoir de ne pas être méchant » ${ }^{71}$.

Il est vrai qu'il peut exister un écart entre l'évaluation qu'un critique accepte de publier et son opinion personnelle d'une œuvre, écart que plusieurs facteurs expliquent. D'abord, le critique, qui agit à titre de public professionnel, n'a pas les mêmes attentes que ses propres lecteurs. Or on s'attend à ce qu'il tienne compte de la tribune qui l'embauche et de son public ${ }^{72}$. Ensuite, le critique devrait dans l'idéal saisir les œuvres en fonction du projet de l'auteur et, comme nous l'avons dit, du contexte de production, sans les juger par rapport au canon ni à ses préférences personnelles. Pool rappelle que le critique débutant devra sans doute ajuster ses standards pour éviter toute déception :

If they entered the field with high standards, as most of us did, the reality of reviewing comes as a blow. [...] [T]he first thing reviewers will need to do is lower their standards. Their own favorite authors may be Tolstoy or Austen, but they won't be reviewing the works of Tolstoy or Austen; rather they will see books that are mostly far inferior [...]. ${ }^{73}$

Cet ajustement est moins grand en milieu minoritaire, où la question du canon se pose de manière relative; c'est sans doute moins la perspective de découvrir un " chef-d'œuvre universel » (si une telle chose existe!) qui motive un volontaire à devenir critique que la possibilité d'investir sa communauté et d'encourager les productions locales.

Les travers d'une œuvre ne devraient jamais servir à justifier une critique médiocre; sur le plan de la qualité, le maillon de la production et celui de la réception critique sont indépendants. C'est pourtant une explication que servent parfois les critiques, excédés que l’on se plaigne de leur travail :

70. Voir notamment François Paré, op. cit., p. 202.

71. Mathieu Charlebois, "ÉDITO - Pourquoi MMEH publie-t-il des critiques négatives ", Ma mère était hipster, 2014; sur Internet : http://mamereetaithipster. com/2014/03/11/edito-pourquoi-mmeh-publie-t-il-des-critiques-negatives/ (page consultée le 30 décembre 2015).

72. Voir Gail Pool, op. cit., p. 90.

73. Ibid., p. 106. 
Or, il faudrait reconnaître qu'il ne peut y avoir de critique littéraire de qualité s'il n'y a pas une production littéraire de qualité pour l'alimenter. Le critique qui lit semaine après semaine de compendieuses niaiseries, des romans qui s'effondrent au bout de dix pages, des poèmes qui tiennent du grognement, finit par perdre toute mesure. ${ }^{74}$

Transposé au journalisme, l'argument paraît tout à fait farfelu : la qualité d'un reportage n'a rien à voir avec la qualité de la nouvelle rapportée. Évidemment le critique, tout comme l'artiste, peut connaître des ratés. Son travail, tout comme les œuvres d'art, peut faire l'objet d'une critique. Mais il importe alors d'opérer une distinction entre une critique négative et une critique médiocre :

Une mauvaise critique n'est pas une critique qui dit du mal d'une œuvre. Une mauvaise critique est une mauvaise analyse qui ne s'appuie pas sur des connaissances suffisantes, qui ne met pas l'œuvre en contexte, qui se contente de résumer ou de porter un jugement binaire sur la forme « j'aime / je n'aime pas $» .75$

Une œuvre de moins bonne qualité ne donne pas raison à un critique de mal effectuer son travail. S'il est vrai qu'elle complique l'écriture d'une recension, une telle œuvre donne plus de latitude au critique pour développer une réflexion originale. Cette réflexion sera d'autant plus pertinente qu'elle pourra peut-être stimuler davantage la production artistique qu'une critique positive. C'est pour cette raison que la qualité des œuvres ne devrait pas non plus servir à justifier l'absence de critique, comme le laisse entendre Mathieu Wade :

C'est que depuis un bon bout de temps, l'essentiel de la production littéraire acadienne est insipide (lire individualiste, égotiste, narcissique, nombriliste, existentialiste, adolescente). Elle ne bouscule rien. Elle ne revendique rien. Elle ne nous apprend rien. [...] [Lorsque l'art] se retire du monde pour ne parler que de l'individu désaffilié et de ses angoisses existentielles, lorsqu'il ne bouscule pas notre rapport à nous-mêmes, à nos préjugés, à la société que nous habitons, qu'on ne s'étonne pas qu'il ne suscite pas de critique... $^{76}$

74. Robert Melançon, « Ne tirez pas sur la critique », Liberté, vol. 23, $\mathrm{n}^{\circ}$ 2, 1981, p. 84.

75. Catherine Voyer-Léger, Métier critique, op. cit., p. 22.

76. Mathieu Wade, op. cit., en ligne. 
Non seulement l'essentiel de la production littéraire acadienne n'est pas telle que la décrit Wade, mais ce sont plutôt les autres enjeux soulevés - l'absence de volontaires, leur impression de ne pas être suffisamment formés, les conditions de travail du critique, la proximité du milieu, la peur d'une réaction négative, etc. - qui expliquent la difficulté de maintenir un espace critique dynamique et continu.

\section{Conclusion}

Parmi ses toutes premières publications, le journal satirique acadien La Plaise faisait paraître, à l'hiver 2015, un billet intitulé "Critique défavorable publiée, la communauté artistique est en émoi ». L'auteur, qui adopte le pseudonyme "Ginette Aucoin-Surette », imagine les réactions de la communauté acadienne face à la critique particulièrement négative d'une (fausse) pièce intitulée Haria à Val-Comeau, dont l'un des artistes serait un certain « Herménégilde C. » :

Une page Facebook intitulée «NON à la critique en Acadie!! »
comprend déjà plus de 3500 abonnés. Parmi les messages,
plusieurs spectateurs de la pièce viennent à sa défense :
"Honnêtement, je l'avoue, la pièce n'était pas très bonne.
Mais de l'écrire dans le journal, ça c'est vraiment dépasser
la ligne », écrit Nadine Chiasson. Pierre Girard, un autre
spectateur, affirme pour sa part que "C'est pas parce que
c'était mauvais qu'on a besoin de l'écrire! »77

En faisant de ces idées reçues la matière d'une parodie, l'article montre combien le discours sur la critique artistique tend à tourner en rond. En Acadie, la discussion sur la place de la critique artistique ou, si on veut, la "critique de la critique » - qu'elle soit dirigée vers le contenu ou l'inexistence du discours critique, ou encore vers la qualité des œuvres acadiennes - est devenue une sorte de réflexe, pour ne pas dire un poncif. Toutefois, le discours public peine à analyser le problème autrement qu'en surface et se méprend souvent sur ses sources véritables.

Afin de briser ce cycle une fois pour toutes et de manière à faire progresser la critique en Acadie, il importerait d'investir de manière égale tous les aspects de la réception. C'est dire qu'il faut miser non seulement sur la critique immédiate, en créant des espaces médiatiques durables qui lui sont destinés et en encadrant les volontaires qui désirent en faire,

77. Ginette Aucoin-Surette (pseudonyme), « Critique défavorable publiée, la communauté artistique est en émoi ", La Plaise, 2 février 2015; sur Internet : https://laplaise.wordpress.com/2015/02/02/critique-defavorable-publiee-la-com munaute-artistique-est-en-emoi/ (page consultée le 30 décembre 2015). 
mais aussi sur les prix, l'enseignement et le public. Tous ces aspects de la réception présentent des défis en Acadie. S'il existe des prix visant à souligner l'excellence artistique, la liste des lauréats est tout aussi périodiquement remise en question que la critique artistique. Notamment, Benoit Doyon-Gosselin a montré que les processus d'attribution des prix littéraires sont parfois contestables et que la légitimité même de ces prix est variable ${ }^{78}$. Pour ce qui est de l'enseignement, le palier universitaire accorde certes une place importante aux œuvres acadiennes, mais ce n'est toujours pas le cas des paliers primaire et secondaire ${ }^{79}$. Enfin, il importe de travailler à développer le public, notamment en déboulonnant certains préjugés qui perdurent par rapport aux arts en milieu minoritaire, de même qu'en augmentant la visibilité et l'accessibilité aux productions artistiques.

Or une tradition de critique artistique durable et bien établie permettrait peut-être de résoudre certains problèmes associés à ces autres facettes de la réception. Par exemple, elle pourrait dégager les prix de la responsabilité de "confirmer l'existence et la vitalité de l'institution $»^{80}$ - ce qu'ils tendent à faire en milieu minoritaire - puisque la critique s'en serait déjà chargée, en recensant de manière adéquate les œuvres artistiques. Les prix pourraient ainsi reprendre leur véritable fonction, c'est-à-dire ne consacrer que les œuvres les plus méritantes. De même, une telle critique pourrait contribuer à outiller les enseignants pour qu'ils abordent davantage les œuvres d'art acadiennes en salle de classe, tout particulièrement au niveau secondaire. Enfin, la critique est un outil incontournable pour inciter le public à découvrir plus régulièrement et mieux apprécier la culture locale. En effet, qui est plus apte à leur servir guide afin de faire ressortir la pertinence de notre art, si ce n'est le critique?

\footnotetext{
78. Voir Benoit Doyon-Gosselin, op. cit.

79. Concernant l'enseignement de la littérature acadienne, voir David Lonergan "Poésie acadienne et institution : de 1990 à aujourd'hui », dans Robert Yergeau (dir.), Itinéraires de la poésie - Enjeux actuels en Acadie, en Ontario et dans l'Ouest canadien, Ottawa, Le Nordir, coll. «Roger-Bernard », 2004, p. 75.

8o. François Paré, op. cit., p. 118.
} 